Pathologe 2014 · [Suppl 2] · 35:188-188

DOI 10.1007/s00292-014-1974-3

Online publiziert: 15. November 2014

(c) Springer-Verlag Berlin Heidelberg 2014
W.D. Travis

Department of Pathology, Department of Cancer Biology \& Genetics,

Memorial Sloan Kettering Cancer Center, NY

\title{
The 2015 WHO classification of lung tumors
}

Largely driven by the molecular revolution and therapeutic breakthroughs, the pathologic diagnosis of lung cancer has been transformed over the past decade. The revision of the 2004 WHO Classification of Lung Tumors is being prepared and should be published in 2015 .

In 2013, the WHO commissioned a panel of editors and an international multidisciplinary panel of authors to develop this classification. During the course of the past year, an international panel of multidisciplinary experts was convened by the International Association for the Study of Lung Cancer (IASLC) and the WHO for a series of meetings to develop this classification. The many advances in clinical, radiologic, histologic and genetic aspects of lung cancer that have been seen during the past decade were reviewed and now provide the basis for this revision.

A major change is the introduction of immunohistochemistry and genetic testing (EGFR mutation and ALK rearrangement) for many tumors, to guide clinicians in making personalized therapeutic decisions. These changes impact not only the evaluation of tumors obtained as nonresection specimens in patients with advanced lung cancer, but also tumors obtained as resection specimens. For adenocarcinoma, the IASLC/American Thoracic Society/European Respiratory Society (IASLC/ATS/ERS) lung adenocarcinoma classification, including the approach to classification in nonresection specimens, was adopted. Unlike previous WHO classifications, the order of the tumors begins with the most common tumor (i.e. invasive adeno- carcinoma) and ends with the preinvasive lesion (i.e. atypical adenomatous hyperplasia). For squamous cell carcinoma, the former "clear cell", "small cell" and"papillary" subtypes were dropped and replaced by the "keratinizing" and "nonkeratinizing" subtypes, with preservation of the "basaloid" subtype. Neuroendocrine tumors are now put into one group. Large cell carcinoma subtypes from the $2004 \mathrm{WHO}$ classification have been discontinued and now this category includes undifferentiated tumors only. The former large cell carcinomas that are pneumocyte marker- or mucinpositive are moved into adenocarcinoma, and the squamous marker-positive cases are reclassified as nonkeratinizing squamous cell carcinoma. These changes are supported by a combination of clinicalpathologic studies, as well as multiple genetic data including recent comprehensive analyses of lung cancer genome alterations. The landscape of lung cancer pathology and classification has been dramatically altered over the past decade, and is now driven by the principles of personalized medicine and genetics.

\section{Corresponding address}

\section{W.D. Travis}

Department of Pathology, Department of Cancer Biology \& Genetics, Memorial Sloan Kettering Cancer Center

1275 York Ave, Room A516, 10065 NY USA

travisw@mskcc.org

\section{Compliance with \\ ethical guidelines}

Conflict of interest. W.D. Travis states that there are no conflicts of interest.

The accompanying manuscript does not include studies on humans or animals.

The supplement this article is part of is not sponsored by the industry. 